\title{
WiMAX Backhaul for Environmental Monitoring
}

\author{
Tuomas Nissilä Kostas Pentikousis Ilkka Harjula Jyrki Huusko Mika Rautio \\ VTT Technical Research Centre of Finland \\ Kaitoväylä 1, FI-90571 Oulu, FINLAND \\ firstname.lastname@vtt.fi
}

\begin{abstract}
In environmental monitoring, wirelessly connected sensors form local networks and collect data, which are sent to a central repository for further analysis and permanent storage. In this paper we empirically evaluate the use of fixed WiMAX to backhaul data from an environmental monitoring application. We find that aggregating sensor readings is crucial for taking full advantage of the WiMAX backhaul. We consider application-layer aggregation for scenarios where sensor communication is programmable or at least configurable. For scenarios where sensors cannot be modified, we consider network-layer aggregation using a performance enhancing proxy. Finally, we consider the case where application- and network-layer aggregation are combined. Our results show that without aggregation, goodput is only a small fraction of the maximum achieved in our testbed. Furthermore, we examine the effect of different modulation schemes on performance and quantify in practice the number of wireless sensor nodes that could be served with different modulation and data aggregation combinations.
\end{abstract}

\section{Categories and Subject Descriptors}

C.2.5 [Computer-Communication Networks]: Localand Wide-Area Networks

\section{General Terms}

Experimentation, Measurement, Performance

\section{Keywords}

WiMAX, IEEE 802.16, Testbed, Wireless Sensor Networks

\section{INTRODUCTION}

Environmental monitoring employing wirelessly connected sensor networks is a very active research area. Wireless sensor nodes are as a rule power- and resource-limited and a lot of related work deals with how to address this handicap. Karl and Willig [4] provide a great overview, discussing a wide range of topics, covering both architecture

Permission to make digital or hard copies of all or part of this work for personal or classroom use is granted without fee provided that copies are not made or distributed for profit or commercial advantage and that copies bear this notice and the full citation on the first page. To copy otherwise, or republish, to post on servers or to redistribute to lists, requires prior specific permission and/or a fee. MUM'2008, December 3-5, 2008, Umeå, Sweden.

Copyright 2008 ACM 978-1-60558-192-7 08/12 _..\$5.00. and protocol-related issues. Glisic [3] presents methods for limiting resource use by performing computations to save on communication costs. The coverage area of wireless sensor networks (WSNs) is typically small and a backhaul, based on a different technology than the one used for communication between the nodes in a WSN, is employed. Wide-area wireless data networks such as Mobitex and GPRS [5] have been used for telemetry and alarm systems but their capacity is limited and so is the number of sensors that can be supported. At the same time, the standards for worldwide interoperability for microwave access (WiMAX) have attracted significant interest $[1,2]$. WiMAX adopts IEEE 802.16 specifications and has the potential to change the field of telecommunications, providing cost-effective broadband wireless access to rural and isolated areas, as well as difficult terrains where wired alternatives cannot be used.

We empirically study an environmental monitoring application which backhauls data using a fixed WiMAX subscriber station (SS). We revisit the communication/computation tradeoff and quantify the gains from application- and network level data aggregation discussed in $[4,7]$. We focus on measuring performance only on the uplink (UL) as we anticipate that several SSs could transmit environmental data to a single base station (BS), possibly employing directional antennas. Note that, in general, UL is dimensioned to smaller capacities than the downlink. We measure goodput with different modulation schemes and under excellent conditions, providing an upper bound on what is possible today with commercial off-the-shelf (COTS) fixed WiMAX equipment.

This paper is organized as follows. Section 2 introduces the environmental monitoring application we are interested in and describes our experimental facility and evaluation methodology. Section 3 presents our experimental results and Section 4 concludes this paper.

\section{ENVIRONMENTAL MONITORING}

Picture an environmental monitoring application where several WSNs are distributed over a large area. A fixed WiMAX SS connects the set of WSNs with the central monitoring system (CMS), backhauling all data collected by the sensor nodes possibly in some remote or wild area. Alternatively, the sensor nodes could monitor apartments in a residential area. The monitoring system comprises three main subsystems: (i) a WiMAX cell, (ii) the access service network (ASN), and (iii) an enhanced environmental monitoring framework (EEMF) extended to the WiMAX 


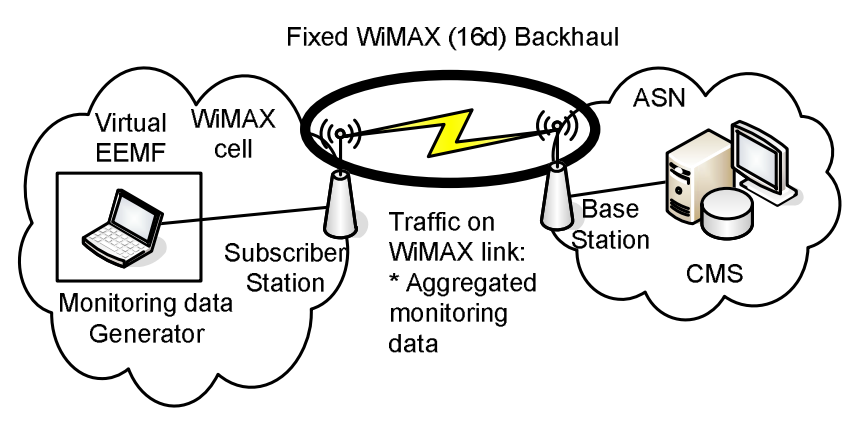

Figure 1: WiMAX testbed schematic

cell. EEMF includes one or more WSN(s), which gather measurements from the monitored field, and separate video surveillance system(s). Each WSN comprises $k$ wireless sensor nodes. Depending on the specific monitoring application, nodes may measure luminous intensity, temperature, or some other interesting field characteristic.

We emulate an environmental monitoring application based on Infineon sensor nodes, developed in the European EYES project. A top priority is to serve large numbers of monitoring nodes while optimizing the backhaul use. Maximizing application-layer throughput, or goodput, necessitates the use of the best possible modulation and avoiding resource waste. Sensor data are typically small in size and transmitting a single reading introduces high overhead into the system. Although IEEE 802.16 does specify SDU and PDU aggregation, and even makes provisions for robust header compression (ROHC) at the link layer, this is not a mandatory feature. In fact, our testbed equipment does not implement any of these features. Thus, there is motivation to aggregate data at protocol layers above the link layer. Clearly, the sensor data transfer direction is from the WiMAX SS to the BS (UL). In this paper, we focus on the aggregation benefits over the WiMAX UL using UDP, which does not provide reliable transmission. Still, sensor readings are often redundant and missing a few measurements, if packet drops occur, is not by and large harmful.

\subsection{Testbed and Experiment Description}

Fig. 1 illustrates the experimental facility used in our empirical performance evaluation study. At the center of it lies an Airspan MicroMAX-SoC fixed WiMAX BS and an Airspan ProST SS (see Table 1 for configuration parameters and $[6,7])$. Symmetrically on the BS and SS side, we connect GNU/Linux (kernel ver. 2.6.20-15, Ubuntu 7.04) PCs with Broadcom NetXtreme BCM5754 1 Gbps Ethernet PCI cards. The tests were performed in a laboratory environment, where conditions were relatively static, even though there may be some light variation in a wireless link. Throughout the experiments we monitored conditions so that we do not deviate under no circumstances from the parameters set in Table 1. We use JTG, an open source network traffic generator, to transmit the emulated sensor data traffic over the WiMAX UL, based on the output of real Infineon sensors, carrying readings of luminous intensity.

Besides experimenting with different aggregation schemes, described in the following subsection, we also evaluated all
Table 1: Testbed Configuration

\begin{tabular}{lc}
\hline \hline PHY & WiMAX 16d, 256 OFDM FDD \\
Frequency band & $3.5 \mathrm{GHz}$ \\
Channel bandwidth & $3.5 \mathrm{MHz}$ \\
UL Modulation & BPSK $1 / 2, \mathrm{QPSK} 3 / 4$ \\
& 16QAM 3/4, 64QAM 3/4 \\
SS-BS distance & 10 m (Line of Sight) \\
SS-BS transmission & $1.0 \mathrm{dBm}\left(60^{\circ}\right.$ directional antenna) \\
MAC scheduling & Best Effort \\
\hline
\end{tabular}

modulations available for our WiMAX UL, namely BPSK, QPSK, 16QAM and 64QAM, see Table 1. To the best of our knowledge this is the first paper to provide such detailed results using a real WiMAX testbed and considering both alternative modulation schemes and different packet sizes. We effectively create two-dimensional matrices that present clear evidence of the benefits of sensor reading aggregation under different modulation schemes, which in real-world deployments correspond to different link conditions. This allows us to analyze the results from different perspectives, serving the needs of a large set of monitoring applications.

The first set for experiments studies application-layer aggregation at the WSN gateway (WSN-GW). The second set studies network-layer aggregation at the performance enhancing proxy (PEP) right before transmission over the $\mathrm{Wi}$ MAX link and the third set studies combinations of application and network-layer aggregation.

\subsection{Monitoring Data Aggregation}

This section provides the necessary background on monitoring data aggregation to assist in understanding the forthcoming results section. By default, a single sensor reading (sensor data) is encapsulated in a single packet. In our chosen application example, a reading corresponds to only 28 bytes/packet. With such a small application payload, the UDP/IP header overhead is $100 \%$; one would expect significant gains by employing aggregation at the application layer. Network-layer aggregation should also be beneficial, but perhaps not be as effective, as the PEP UDP/IP headers add another 28 bytes to the total aggregated packet size.

Fig. 2 illustrates the aggregated packet flow from a sensor node via the WiMAX cell towards CMS. Aggregation is performed in two steps. The first step is application-layer aggregation at WSN-GW. The second step is network-layer aggregation by the PEP. Packets are then transmitted in SS-BS UL subframes, and follow their path from the BS to the CMS. An aggregated monitoring packet carries two or more sensor readings. We experimented with varying levels of application-layer aggregation, concatenating from 1 to 52 sensor readings, i.e. samples (S), in a single IP packet, and network-layer aggregation (from 1 to 26 sensor readings). For the combination of the two, we chose some optimal application and network aggregation levels.

Fig. 2 shows that each sensor generates and transmits a 28-byte packet per sample time, $t_{s}$. That is, $M$ packets arrive at the WSN-GW every $t_{s}$. When application-layer aggregation is employed at the WSN-GW, several readings are packetized together. The WSN-GW forwards packets to PEP, where network-layer aggregation optionally takes place. The PEP can aggregate packets from $N$ different 


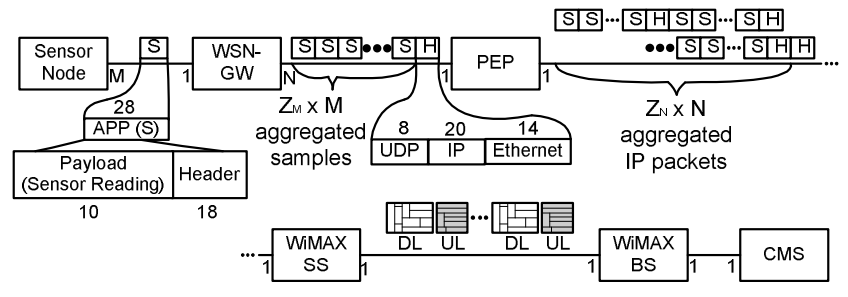

Figure 2: Aggregated packet flow towards the CMS

flows. In a hybrid case, application-layer aggregation at WSN-GW is followed by network-layer aggregation at the PEP. The maximum combined level of aggregation in $t_{s}$ is $M \times N$, which means that $N$ IP packets, each containing $M$ sensor readings, are aggregated together. If higher aggregation levels are desired, readings for multiples of $t_{s}$ must be buffered. The packet inter-departure time $T$ at the PEP is

$$
T=\left(Z_{M} \times Z_{N}\right) t_{s}+t_{e},
$$

where $Z_{M}, Z_{N} \in \mathbb{N}$, and $t_{e}$ the time window of the arriving packets from the WSN-GW(s). Increasing $T$ decreases the frequency of data arrivals at the CMS roughly by $\left(Z_{M N}=\right.$ $Z_{M} \times Z_{N}$ ). This might be undesirable for some applications, and can be addressed by decreasing the sampling time.

An important issue is the overall delay, $D_{M, N}$, between the time that a sensor reading is transmissted from the WSN till it is received by the CMS. This can be expressed as

$$
D_{M, N}=T+d_{p},
$$

where $d_{p}$ is a sum of all delays in the path, comprising mainly link/network delays. Thus, given the sampling time, the path delays and the desired aggregation levels, the overall delay $D_{M, N}$ can be calculated. For many monitoring applications, sensor readings are received only once per second, or even less frequently, and aggregation makes a lot of sense. Other applications need real-time measurements and the decision of whether to use aggregation and, if so, which aggregation level to employ is an optimization problem that depends on the application QoS requirements. In real-world deployments, networkers have to strike a balance between the overall delay $D_{M, N}$ and the gains from aggregation.

For some monitoring applications, heuristic addressing can be adopted as proposed in [4]. Using sensor IDs, CMS can map each received reading to the originating sensor node. In this case, the PEP could compress the UDP/IP headers into a single ID field, decreasing header overhead significantly in the case of network-layer aggregation. However, this is not studied here and is left for future work.

\section{RESULTS}

As we are interested in quantifying the number of sensor nodes that can be served by a single COTS WiMAX SS, using its UL to backhaul readings to CMS, we choose application layer throughput, also referred to as goodput, as our primary metric. Recall that we experiment with both different levels of sensor reading aggregation and different modulation schemes, producing a full matrix of results.

We made five independent runs for each configuration, each lasting 300 seconds, to get a reasonable performance estimate within each measurement run. The results below present the median values over the five measurement runs.

We first performed baseline experiments to determine the maximum goodput that can be attained in our testbed with different modulation schemes. We saturated the fixed WiMAX UL and measured the maximum goodput. We experimented with various UDP packet sizes and obtained the best results for application payloads of 1472 bytes (Maximum Transmission Unit or MTU $=1500$ bytes, which is the recommended MTU size for IEEE 802.16 standard-compliant equipment): The average maximum measured goodput was $5.52 \mathrm{Mb} / \mathrm{s}$ with 64QAM, $3.76 \mathrm{Mb} / \mathrm{s}$ with 16QAM, $1.48 \mathrm{Mb} / \mathrm{s}$ with QPSK, and only $0.51 \mathrm{Mb} / \mathrm{s}$ with BPSK. When no sensor reading aggregation is employed and a single sensor reading is transmitted in a single UDP datagram the measured goodput in our testbed was $1.07 \mathrm{Mb} / \mathrm{s}$ for 64QAM, 1.07 $\mathrm{Mb} / \mathrm{s}$ for 16QAM, $0.57 \mathrm{Mb} / \mathrm{s}$ for QPSK, and only $0.20 \mathrm{Mb} / \mathrm{s}$ with BPSK. These measurements serve as a reference in the remainder.

Fig. 3 (left) presents the measurement results when application layer aggregation is employed. Base 10 logarithmic scale is used for the vertical axis. Application-layer aggregation improves goodput significantly irrespective of the modulation scheme. Relatively large gains can be achieved even when employing rather low aggregation levels $(M<6)$. Although the higher the application-layer aggregation level the better, this is a diminishing gains process. And, one has always to consider the tradeoff of delaying samples to achieve better utilization of the WiMAX backhaul. With $30 \leq M \leq 52$, cumulative goodput is close to the highest achievable goodput, i.e. when using MTU-sized packets. When the best modulation scheme is used alongside the highest aggregation level, more than an order of magnitude increase in goodput can be achieved.

Fig. 3 (center) presents our measurement results for networklayer aggregation. In this case, no application-layer aggregation is employed, and it is the responsibility of the PEP to make the most of the WiMAX UL by aggregating singlereading sensor packets into a larger packet. We reiterate that in both network- and application-layer aggregation the solution is based solely on software. No support from the underlying WiMAX hardware is necessary. The PEP, however, may be acting in close cooperation with a router. In contrast with the application-layer aggregation where readings from a single sensor node are put together in a packet, the PEP in network-layer aggregation will often put in the same aggregated packet packets carrying readings from different nodes, and possibly from different WSNs. As Fig. 3 illustrates, network-layer aggregation offers tangible benefits in terms of goodput, although not as high as application-layer aggregation. This is mainly due to the increased header overhead as less of the payload of each packet backhauled over WiMAX is carrying sensor reading data. When $N=2,3$, there is a significant gain in goodput for 16QAM and 64QAM. After that, the goodput increase is clear but not as impressive. Due to MTU restrictions we cannot aggregate more the $M=26$ readings.

We also explored the case where both application and net- 

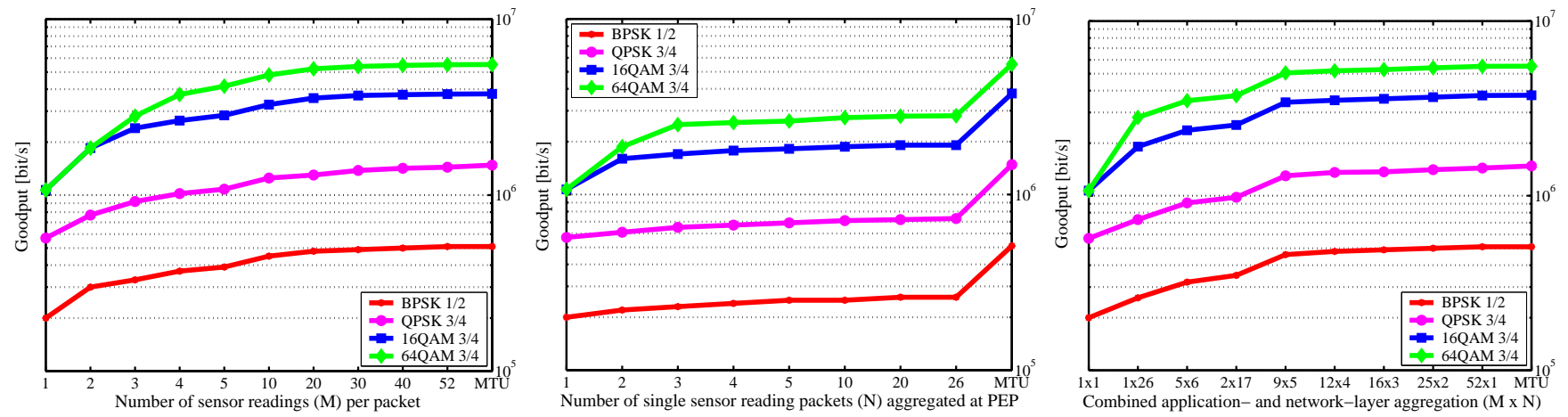

Figure 3: Application-layer (left), network-layer (center), and combined (right) aggregation results

work layer aggregation are employed. This would be the case when sensor readings are aggregated first at the WSN-GW and, further on, flows from possibly different WSN-GW(s) are aggregated at the PEP, before being backhauled over the WiMAX UL. Fig. 3 (right) presents the results from our WiMAX testbed for this combined aggregation experiments. As before, the baseline results are presented for ease of comparison. Although at first sight the exact combinations we experimented with may appear as arbitrary, they in fact consider specific scenarios, and indicate remarkable goodput gains. The highest network-layer aggregation ( $M=1, N=26$ ) allows, roughly, for about half of the MTU goodput. Comparing that to other results, the effectiveness of application-layer aggregation is clearly seen. The $M=5, N=6$ scheme leads to a less than optimal packet size. For $M>10$ goodput gains taper off. Overall, $M=9, N=5$ appears to be a good compromise between complexity, sample delivery delay, and goodput gains but this is clearly specific to the application scenario. Extensive application-only aggregation with minimal $(M=25, N=2)$ or no $(M=52)$ network-layer aggregation is the winner in terms of absolute goodput gains but, as discussed, it may be unrealistic to introduce large buffering delays in sensor reading delivery for many environmental applications.

The environmental monitoring measurement results presented in this section are, to the best of our knowledge, the first publicly reported measurements of this kind in the literature. Although analysis, modeling, and simulation could have provided similar insights, we argue that it is important to measure and validate what COTS WiMAX equipment can deliver in practice. Many a projects have failed because of overestimating the capabilities of real-world implementations of otherwise well-established standards. WiMAX performance in practice is still not well-studied and we hope that this paper provides insights for other researchers.

\section{CONCLUSION}

Despite the significant interest and developments in WiMAX technology, WiMAX equipment is still expensive and out or reach for most researchers. As a result, most WiMAXrelated studies are performed using simulation and modeling, or solely analytical work. Few studies present real measurements from testbeds or field tests. We described an environmental monitoring system, which uses fixed WiMAX to backhaul sensor readings to a central repository. We studied its performance using synthetically generated traffic and quantified the gains from sensor reading aggregation. We considered application- and network-layer aggregation, and a hybrid aggregation scheme for the monitoring data transfer in our testbed, and measured goodput over the whole range of implemented modulations, from BPSK to 64QAM.

Our results indicate how WiMAX deployments can benefit from aggregation. The results show that relatively similar gains can be achieved with all WiMAX UL modulations used in our experiments. Even with lower aggregation levels the gains are significant. When using basic aggregation methods, the application-layer aggregation gains were larger than the respective network-layer aggregation gains. However, for sensor data aggregation at the network level, the IP and UDP headers could be compressed into a smaller ID-based header. This could lead network aggregation results closer to the ones reported for application aggregation. Using the minimum amount of bits to represent the sensor readings with respect to the required accuracy, the sensor samples could be optimized to bring additional performance gains for demanding applications.

\section{REFERENCES}

[1] J. G. Andrews, A. Ghosh, and R. Muhamed. Fundamentals of WiMAX. Prentice Hall, March 2007.

[2] A. Ghosh, D. Wolter, J. Andrews, and R. Chen. Broadband wireless access with WiMax/802.16: current performance benchmarks and future potential. IEEE Communications Magazine, 43(2):129-136, Feb. 2005.

[3] S. G. Glisic. Advanced Wireless Networks, 4G Technologies. J. Wiley \& Sons, West Sussex, UK, 2006.

[4] H. Karl and A. Willig. Protocols and Architectures for Wireless Sensor Networks. J. Wiley \& Sons, West Sussex, UK, July 2007.

[5] K. Pentikousis. Wireless data networks. The Internet Protocol Journal, 8(1):6-14, 2005.

[6] K. Pentikousis, J. Pinola, E. Piri, and F. Fitzek. An experimental investigation of VoIP and video streaming over fixed WiMAX. In Proc. 4th Intl. Workshop on Wireless Network Measurements (WiNMee), Berlin, Germany, March 2008.

[7] K. Pentikousis, E. Piri, J. Pinola, F. Fitzek, T. Nissilä, and I. Harjula. Empirical evaluation of VoIP aggregation over a fixed WiMAX testbed. In Proc. 4 th Intl. Conf. on Testbeds and Research Infrastructures for the development of networks $\& 5$ communities (TridentCom), Innsbruck, Austria, March 2008. 